\title{
OWA-FRPS: A Prototype Selection method based on Ordered Weighted Average Fuzzy Rough Set Theory
}

\author{
Nele Verbiest ${ }^{1}$, Chris $_{\text {Cornelis }}{ }^{1,2}$, and Francisco Herrera ${ }^{2}$ \\ 1 Department of Applied Mathematics, Computer Science and Statistics, Ghent \\ University, Krijgslaan 281 (S9), B-9000 Gent, Belgium \\ Nele.Verbiest@UGent . be \\ 2 Department of Computer Science and Artificial Intelligence, University of Granada, \\ Calle del Periodista Daniel Saucedo Aranda s/n, 18071 Granada, Spain \\ chriscornelis@ugr.es herrera@decsai.ugr.es
}

\begin{abstract}
The Nearest Neighbor (NN) algorithm is a well-known and effective classification algorithm. Prototype Selection (PS), which provides NN with a good training set to pick its neighbors from, is an important topic as $\mathrm{NN}$ is highly susceptible to noisy data. Accurate state-of-the-art PS methods are generally slow, which motivates us to propose a new PS method, called OWA-FRPS. Based on the Ordered Weighted Average (OWA) fuzzy rough set model, we express the quality of instances, and use a wrapper approach to decide which instances to select. An experimental evaluation shows that OWA-FRPS is significantly more accurate than state-of-the-art PS methods without requiring a high computational cost.
\end{abstract}

Keywords: Ordered Weighted Average, Fuzzy Rough Sets, Prototype Selection, KNN

\section{Introduction}

One of the most well-known and most widely used classification algorithms is Nearest Neighbors (NN,[1]). This method classifies a test instance $t$ to the class of the nearest neighbor of $t$ in the training set. Although NN has been proven to be very useful for many classification problems, it deals with some problems, among which its sensitivity to noise and its large storage requirements are the most important ones.

In this work we alleviate these problems by using Prototype Selection (PS,[2]). This technique removes redundant and/or noisy instances from the training set, such that the training set requires less storage and such that the NN algorithm is more accurate. PS techniques that mainly try to improve the classification accuracy are called edition methods, those that focus on reducing the required storage are condensation methods. Hybrid PS techniques try to tackle both problems simultaneously. In this work we develop an editing method. Many PS methods have been proposed in the literature, a comprehensive overview 
can be found in [2]. When the algorithm does not make use of a specific classifier to classify the entire training set, the method is called a filter method. Condensation methods do use a specific classifier, the NN classifier in our case, to classify the entire training data to obtain a quality assessment of a certain prototype subset. Filter methods are generally faster and less accurate, while wrapper methods are slower and more accurate. Many wrapper PS algorithms are evolutionary based, like CHC [3], GGA [4,5] or SSMA [6], while others use other search heuristics like RMHC [7] or RNG [8]. Most of the filter methods are based on the NN algorithm itself, like AllKNN [9] or MENN [10]. The method that we develop is a wrapper.

Although many researchers have focused on developing fuzzy rough feature selection [11] algorithms, there is not much literature on fuzzy rough PS yet. Nevertheless, fuzzy rough set theory [12] is a good tool to model noisy data. To the best of our knowledge, the only fuzzy rough based PS method is FRIS [13]. This method selects those instances that have a fuzzy positive region higher than a certain threshold. This method has some problems, the main one being that the method's performance highly relies on a good threshold selection.

In this work, we propose a new fuzzy rough based PS method that assesses the quality of instances using Ordered Weighted Average (OWA) fuzzy rough set theory [14], a more robust version of fuzzy rough set theory, and automatically selects an appropriate threshold.

The remainder of this work is structured as follows. In Section 2, we first discuss three OWA fuzzy rough quality measures that can be used to assess the quality of instances, and then show how these measures can be used to carry out PS. In Section 3, we evaluate our algorithm, called OWA Fuzzy Rough Prototype Selection (OWA-FRPS), and we conclude in Section 4.

\section{Ordered Weighted Average based Fuzzy Rough Prototype Selection}

In this section we present our new PS method. In the first subsection we define three measures to assess the quality of instances, and in the second subsection we demonstrate how we can use these measures to carry out PS.

\subsection{Assessing the quality of instances using OWA fuzzy rough sets}

First we introduce some notations. We consider a decision system $(X, \mathcal{A} \cup\{d\})$, consisting of $n$ instances $X=\left\{x_{1}, \ldots, x_{n}\right\}, m$ attributes $\mathcal{A}=\left\{a_{1}, \ldots, a_{m}\right\}$ and a decision attribute $d \notin \mathcal{A}$. We denote by $a(x)$ the value of an instance $x \in X$ for an attribute $a \in \mathcal{A}$. We assume that each continuous attribute $a \in \mathcal{A}$ is normalized, that is, $\forall x \in X: a(x) \in[0,1]$. The categorical attributes can take values in a finite set. The decision attribute $d$ is categorical too and assigns a class $d(x)$ to each instance $x \in X$.

We associate a fuzzy indiscernibility relation $R: X \times X \rightarrow[0,1]$ with the decision system as follows. First, we calculate the fuzzy indiscernibility $R_{a}$ for each feature 
$a \in \mathcal{A}$ separately. When $a$ is categorical, $R_{a}(x, y)=1$ for $x, y \in X$ if $a(x)=a(y)$ and $R_{a}(x, y)=0$ otherwise. When $a$ is continuous, $R_{a}(x, y)=1-|a(x)-a(y)|$ for all $x, y \in X$.

Next, we combine these separate fuzzy indiscernibility relations using a t-norm $\mathcal{T}$ (the Lukasiewicz t-norm ${ }^{3}$ in this paper):

$$
\forall x, y \in X: R(x, y)=\underbrace{\mathcal{T}\left(R_{a}(x, y)\right)}_{a \in \mathcal{A}}
$$

This fuzzy indiscernibility relation is the keystone of fuzzy rough set theory. A fuzzy set $S$ can be approximated by its fuzzy rough lower approximation

$$
\forall x \in X:(R \downarrow S)(x)=\min _{y \in X} \mathcal{I}(R(x, y), S(y))
$$

with $\mathcal{I}$ the Lukasiewicz implicator ${ }^{4}$ in this paper, and by its upper approximation

$$
\forall x \in X:(R \uparrow S)(x)=\max _{y \in X} \mathcal{T}(R(x, y), S(y))
$$

The fuzzy lower approximation expresses to what extent instances similar to $x$ also belong to $S$, while the upper approximation expresses to what extent there exist instances that are similar to $x$ and belong to $S$.

These concepts can be used to assess the quality of instances. First, note that we can consider the class $[x]_{d}$ of an instance $x \in X$ as a fuzzy set in $X$ :

$$
\forall y \in X:[x]_{d}(y)= \begin{cases}1 & \text { if } d(x)=d(y) \\ 0 & \text { else }\end{cases}
$$

which can be considered as the crisp set that contains all instances that have the same class as $x$.

If we want to assess the quality of an instance $x$, we can use the lower approximation of $[x]_{d}$ :

$$
\left(R \downarrow[x]_{d}\right)(x) .
$$

This value expresses to what extent instances similar to $x$ also belong to the same class as $x$. Another option is to use the upper approximation of $[x]_{d}$ :

$$
\left(R \uparrow[x]_{d}\right)(x)
$$

which expresses to what extent there exist instances that are similar to $x$ and that belong to the same class as $x$.

Both measures are particularly meaningful in the context of NN classification, because they rate instances highly if they are surrounded by neighbors of the same class: the lower approximation measure is high for $x$ if there are no instances

\footnotetext{
3 The Lukasiewicz t-norm is the mapping $\mathcal{T}:[0,1]^{2} \rightarrow[0,1]$, such that $\forall a, b \in$ $[0,1], \mathcal{T}(a, b)=\max (0, a+b-1)$

4 The Lukasiewicz implicator is the mapping $\mathcal{I}:[0,1]^{2} \rightarrow[0,1]$, such that $\forall a, b \in$ $[0,1], \mathcal{I}(a, b)=\min (1-a+b, 1)$
} 
from a different class that are near (similar) to $x$, while the upper approximation measure is high if there exist neighbors from the same class.

In [14] it was noted that the traditional fuzzy rough approximations are highly susceptible to noise, as they use the crisp min and max operators, such that single instances can drastically influence the approximation values. A solution to this problem is to use OWA fuzzy rough sets [14], which replace these crisp operators by softer OWA operators [15]. Recall that, given a weight vector $W=\left\langle w_{1}, \ldots, w_{n}\right\rangle$ for which $\sum_{i=1}^{n} w_{i}=1$ and $\forall i \in 1, \ldots, n, w_{i} \in[0,1]$, the OWA aggregation of $n$ values $s_{1}, \ldots, s_{n}$ is given by:

$$
O W A_{W}\left(s_{1}, \ldots, s_{n}\right)=\sum_{i=1}^{n} w_{i} t_{i}
$$

where $t_{i}=s_{j}$ if $s_{j}$ is the $i$ th largest value in $s_{1}, \ldots, s_{n}$.

When $\langle 0, \ldots, 0,1\rangle$ is used as weight vector, the minimum operator is retrieved, which is the operator that is used in the traditional fuzzy lower approximation. We replace this minimum by a less strict operator that still has the characteristics of a minimum operator, that is, we consider a weight vector with ascending weights, such that lower values get higher weights, and higher values get lower weights. In this work we use the weight vector $W_{\min }=\left\langle w_{1}, \ldots, w_{n}\right\rangle$ where

$$
\forall i \in 1, \ldots, n: w_{i}=\frac{i}{n(n+1) / 2} .
$$

Completely analogously, we can define the $O W A_{W_{\max }}$ operator that softens the maximum operator. Its weights $W_{\max }=\left\langle w_{1}, \ldots, w_{n}\right\rangle$ are defined as follows in this paper:

$$
\forall i \in 1, \ldots, n: w_{i}=\frac{n-i+1}{n(n+1) / 2} .
$$

Replacing the strict minimum and maximum operators in the traditional definitions of fuzzy lower and upper approximation leads to the following more robust definitions of OWA fuzzy rough sets:

$$
\begin{aligned}
& \forall x \in X:\left(R \downarrow^{O W A} S\right)(x)=O \underset{y \in X}{A_{W_{\min }}} \mathcal{I}(R(x, y), S(y)) \\
& \forall x \in X:\left(R \uparrow{ }^{O W A} S\right)(x)=O \underset{y \in X}{W A_{W_{\max }}} \mathcal{T}(R(x, y), S(y))
\end{aligned}
$$

We will use this OWA fuzzy rough set model, leading to the following three quality measures:

$$
\begin{aligned}
& \forall x \in X: \gamma_{L}(x)=\left(R \downarrow^{O W A}[x]_{d}\right)(x), \\
& \forall x \in X: \gamma_{U}(x)=\left(R \uparrow O W A[x]_{d}\right)(x),
\end{aligned}
$$

and

$$
\forall x \in X: \gamma_{L U}(x)=\left(R \downarrow^{O W A}[x]_{d}\right)(x)+\left(R \uparrow O W A[x]_{d}\right)(x)
$$




\subsection{OWA-FRPS}

Based on the quality measures $\gamma$ defined in the previous subsection, we can formulate an algorithm to find a good subset of instances. We obviously want to select the instances with a high $\gamma$ value and remove those with a low $\gamma$ value, but now the question raises what threshold to use.

The main idea of our approach is to use the $\gamma$ values of all instances in $X$ as threshold. We calculate the leave-one-out training accuracy of the corresponding reduced subsets of instances and select the threshold that corresponds to the highest accuracy. More specifically, we carry out the following steps:

1. Calculate the $\gamma(x)$ values for all instances $x \in X$.

2. Remove the duplicates among all these $\gamma$ values, the final set of $\gamma$ values, which will all be considered as thresholds, is $G=\left\{\tau_{1}, \ldots, \tau_{p}\right\}, p \leq n$.

3. For each of the thresholds $\tau \in G$, consider the following subset: $S_{\tau}=\{x \in$ $X \mid \gamma(x) \geq \tau\}$.

4. Calculate the training leave-one-out accuracy of each of these subsets using the LOO procedure in Algorithm 1.

5. Select the subsets $S_{\tau_{i_{1}}}, \ldots, S_{\tau_{i_{s}}}$ with the highest leave-one-out accuracy. Note that multiple subsets can correspond to the same leave-one-out accuracy.

6. Return the subset $S_{\text {median }\left(\tau_{i_{1}}, \ldots, \tau_{i_{s}}\right)}$.

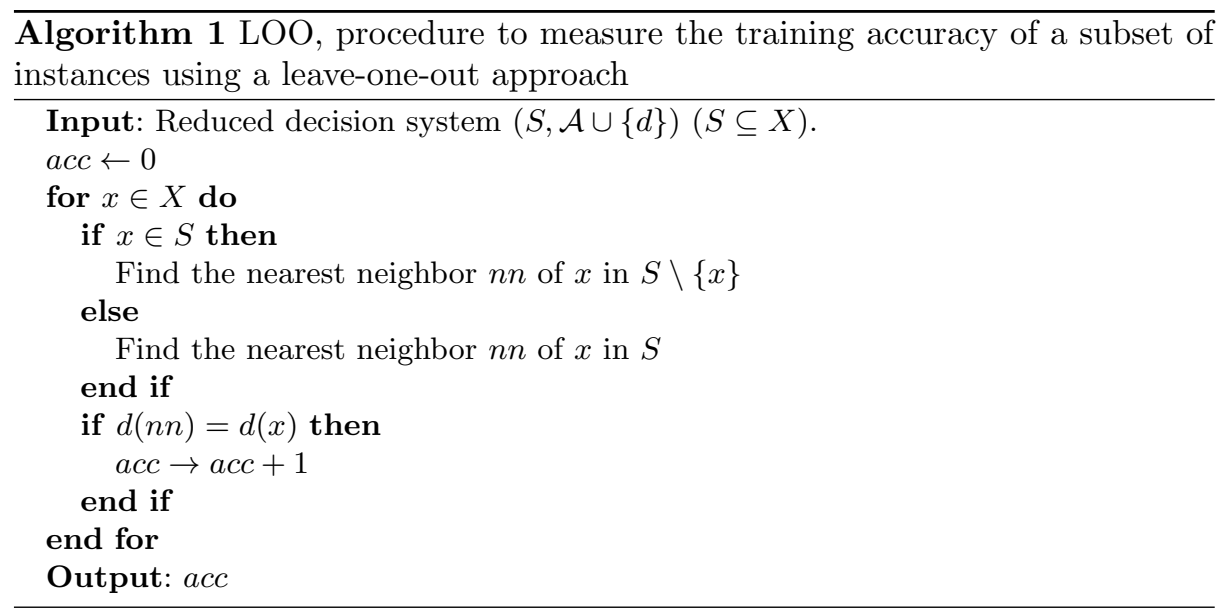

We illustrate the algorithm with an example. Consider the decision system in Table 1, with ten instances, two continuous features and one categorical feature. The values $\gamma_{L U}$ are given in the last column for each instance. There are no duplicates, so the set of thresholds consists of the ten values in the last column of Table 1. In Table 2, we show the corresponding subsets. In order to calculate the training leave-one-out accuracy, we need the Euclidean distances between 
the instances, which are given in Table 3 . In the last two columns of Table 2, the instances that are correctly classified using the subset $S_{\tau}$ are given, together with the training accuracy. The subsets corresponding to the highest LOO training accuracy are $S_{\tau_{1}}, S_{\tau_{3}}$ and $S_{\tau_{9}}$, and subset $S_{\tau_{3}}=\left\{x_{1}, x_{3}, x_{5}, x_{9}\right\}$ will be returned by the OWA-FRPS algorithm.

Table 1. Decision system with 2 continuous features $\left(a_{1}\right.$ and $\left.a_{2}\right)$ and one categorical feature $\left(a_{3}\right)$. The class is given in column $d$ and the value for the $\gamma_{L U}$ measure is shown in the last column.

\begin{tabular}{l|lll|l|l} 
& $a_{1}$ & $a_{2}$ & $a_{3}$ & $\mathrm{~d}$ & $\gamma_{L U}$ \\
\hline$x_{1}$ & 0.2 & 0.4 & $\mathrm{~A}$ & 0 & 1.02 \\
$x_{2}$ & 0.3 & 0.3 & $\mathrm{~A}$ & 1 & 1.016 \\
$x_{3}$ & 1 & 0 & $\mathrm{~B}$ & 0 & 1.16 \\
$x_{4}$ & 0.7 & 0.9 & $\mathrm{~B}$ & 1 & 1.07 \\
$x_{5}$ & 0.4 & 0.3 & $\mathrm{~A}$ & 0 & 1.05 \\
$x_{6}$ & 0.3 & 0.6 & $\mathrm{~A}$ & 1 & 1.01 \\
$x_{7}$ & 0.4 & 1 & $\mathrm{~B}$ & 0 & 1.06 \\
$x_{8}$ & 0.3 & 0.2 & $\mathrm{~B}$ & 1 & 1.15 \\
$x_{9}$ & 0.7 & 0.5 & $\mathrm{~A}$ & 0 & 1.17 \\
$x_{10}$ & 0 & 0.1 & $\mathrm{~A}$ & 1 & 1.14
\end{tabular}

Table 2. Thresholds $\tau$ considered in the OWA-FRPS algorithm and corresponding subsets of instances $S_{\tau}$.

\begin{tabular}{l|l|l|l} 
Threshold $\tau$ & Corresponding subset $S_{\tau}$ & Correctly classified instances & LOO training accuracy \\
\hline 1.02 & $\left\{x_{1}, x_{3}, x_{4}, x_{5}, x_{7}, x_{8}, x_{9}, x_{10}\right\}$ & $\left\{x_{1}, x_{5}, x_{6}, x_{9}, x_{10}\right\}$ & 0.5 \\
1.016 & $\left\{x_{1}, x_{2}, x_{3}, x_{4}, x_{5}, x_{7}, x_{8}, x_{9}, x_{10}\right\}$ & $\left\{x_{5}\right\}$ & 0.1 \\
1.16 & $\left\{x_{3}, x_{9}\right\}$ & $\left\{x_{1}, x_{3}, x_{5}, x_{7}, x_{9}\right\}$ & 0.5 \\
1.07 & $\left\{x_{3}, x_{4}, x_{8}, x_{9}, x_{10}\right\}$ & $\left\{x_{2}, x_{4}, x_{5}\right\}$ & 0.3 \\
1.05 & $\left\{x_{3}, x_{4}, x_{5}, x_{7}, x_{8}, x_{9}, x_{10}\right\}$ & $\left\{x_{4}, x_{5}, x_{9}\right\}$ & 0.3 \\
1.01 & $\left\{x_{1}, x_{2}, x_{3}, x_{4}, x_{5}, x_{6}, x_{7}, x_{8}, x_{9}, x_{10}\right\}$ & $\left\{x_{5}, x_{9}\right\}$ & 0.2 \\
1.06 & $\left\{x_{3}, x_{4}, x_{7}, x_{8}, x_{9}, x_{10}\right\}$ & $\left\{x_{2}, x_{5}\right\}$ & 0.2 \\
1.15 & $\left\{x_{3}, x_{9}, x_{10}\right\}$ & $\left\{x_{3}, x_{5}, x_{7}\right\}$ & 0.3 \\
1.17 & $\left\{x_{9}\right\}$ & $\left\{x_{1}, x_{3}, x_{5}, x_{7}, x_{9}\right\}$ & 0.5 \\
1.14 & $\left\{x_{3}, x_{9}, x_{10}\right\}$ & $\left\{x_{3}, x_{5}, x_{7}\right\}$ & 0.3
\end{tabular}

\section{Experimental Evaluation}

In this section we carry out an experimental evaluation to demonstrate the benefits of OWA-FRPS over other PS methods. 
Table 3. Euclidean distance between the instances

\begin{tabular}{l|llllllllll} 
& $x_{1}$ & $x_{2}$ & $x_{3}$ & $x_{4}$ & $x_{5}$ & $x_{6}$ & $x_{7}$ & $x_{8}$ & $x_{9}$ & $x_{10}$ \\
\hline$x_{1}$ & 0.000 & 0.082 & 0.775 & 0.707 & 0.129 & 0.129 & 0.683 & 0.592 & 0.294 & 0.208 \\
$x_{2}$ & 0.082 & 0.000 & 0.726 & 0.712 & 0.058 & 0.173 & 0.707 & 0.580 & 0.258 & 0.208 \\
$x_{3}$ & 0.775 & 0.726 & 0.000 & 0.548 & 0.695 & 0.785 & 0.673 & 0.420 & 0.668 & 0.819 \\
$x_{4}$ & 0.707 & 0.712 & 0.548 & 0.000 & 0.695 & 0.645 & 0.183 & 0.465 & 0.622 & 0.843 \\
$x_{5}$ & 0.129 & 0.058 & 0.695 & 0.695 & 0.000 & 0.183 & 0.705 & 0.583 & 0.208 & 0.258 \\
$x_{6}$ & 0.129 & 0.173 & 0.785 & 0.645 & 0.183 & 0.000 & 0.624 & 0.622 & 0.238 & 0.337 \\
$x_{7}$ & 0.683 & 0.707 & 0.673 & 0.183 & 0.705 & 0.624 & 0.000 & 0.465 & 0.668 & 0.810 \\
$x_{8}$ & 0.592 & 0.580 & 0.420 & 0.465 & 0.583 & 0.622 & 0.465 & 0.000 & 0.645 & 0.606 \\
$x_{9}$ & 0.294 & 0.258 & 0.668 & 0.622 & 0.208 & 0.238 & 0.668 & 0.645 & 0.000 & 0.465 \\
$x_{10}$ & 0.208 & 0.208 & 0.819 & 0.843 & 0.258 & 0.337 & 0.810 & 0.606 & 0.465 & 0.000
\end{tabular}

\subsection{Experimental Set-up}

We use 28 datasets from the KEEL dataset repository ${ }^{5}$. The characteristics of these datasets are listed in Table 4. As our main focus is to improve the accuracy of NN, we compare OWA-FRPS with 12 PS algorithms that are most accurate according to the study performed in [2]. Additionally, we also compare OWAFRPS to FRIS [13] with parameter value $\alpha=10$. In Table 5 , we give an overview of the algorithms we consider with references to the literature. Note that we use three versions of the new OWA-FRPS algorithm, depending on which measure is used to rank the instances.

For each dataset and PS method, we carry out the following 10 fold cross validation procedure. For each fold, we apply the PS method to the remaining folds (the train data) and then let NN find the nearest neighbors of the test instances in this reduced training set. We report the average classification accuracy, reduction and running time over the 10 folds.

\subsection{Results}

In Table 6, we show the average accuracy, reduction (the percentage of removed instances) and running time (in seconds) over all datasets. First, we note that on average, the OWA-FRPS-LU algorithm is more accurate than the other versions, which shows that both the lower and upper approximation contribute to the quality assessment of the instances. All OWA-FRPS algorithms outperform the state-of-the-art PS algorithms. From now on, we continue the analysis with OWA-FRPS-LU, to which we simply refer to as OWA-FRPS. To test if the improvement is significant, we carry out the statistical Friedman test and Holm post hoc procedure [21]. The Friedman ranks and the adjusted p-values of the Holm post hoc procedure are listed in Table 7. The OWA-FRPS algorithm has the best (i.e. lowest) rank. The low adjusted p-values confirm that OWA-FRPS is significantly more accurate than the state-of-the-art PS algorithms.

\footnotetext{
${ }^{5}$ www.keel.es
} 
Table 4. Datasets used in the experimental evaluation with their number of instances (\#Inst.), number of features (\#Feat.) and number of classes (\#Cl.).

\begin{tabular}{lccc|lccc} 
Name & \#Inst. & \#Feat. \#Cl. & Name & \#Inst. & \#Feat. & \#Cl. \\
\hline appendicitis & 106 & 7 & 2 & housevotes & 232 & 16 & 2 \\
australian & 690 & 14 & 2 & iris & 150 & 4 & 3 \\
automobile & 150 & 25 & 6 & led7digit & 500 & 7 & 10 \\
balance & 625 & 4 & 3 & lymphography & 148 & 18 & 4 \\
bands & 365 & 19 & 2 & mammographic & 830 & 5 & 2 \\
breast & 277 & 9 & 2 & new thyroid & 215 & 5 & 3 \\
bupa & 345 & 6 & 2 & pima & 768 & 8 & 2 \\
crx & 653 & 15 & 2 & saheart & 462 & 9 & 2 \\
dermatology & 358 & 34 & 6 & sonar & 208 & 60 & 2 \\
ecoli & 336 & 7 & 8 & vehicle & 846 & 18 & 4 \\
glass & 214 & 9 & 7 & vowel & 990 & 13 & 11 \\
haberman & 306 & 3 & 2 & wine & 178 & 13 & 3 \\
hayesroth & 160 & 4 & 3 & wisconsin & 683 & 9 & 2 \\
heart & 270 & 13 & 2 & zoo & 101 & 16 & 7
\end{tabular}

Table 5. Overview of the algorithms evaluated in the experimental study.

\begin{tabular}{l|l|c} 
Name & Description & Reference \\
\hline AllKNN & NN based filter method & {$[9]$} \\
CHC & Evolutionary based wrapper method & {$[3]$} \\
GGA & Evolutionary based wrapper method & {$[4,5]$} \\
HMNEI & Hit and miss network based filter method & {$[16]$} \\
MENN & NN based filter method & {$[10]$} \\
ModelCS & Tree-based filter method & {$[17]$} \\
MSS & Spatial-based filter method & {$[18]$} \\
POP & Spatial-based filter method & {$[19]$} \\
RMHC & Random mutation hill climbing wrapper method & {$[7]$} \\
RNG & Graph based wrapper method & {$[8]$} \\
RNN & NN based filter method & {$[20]$} \\
SSMA & Evolutionary wrapper method & {$[6]$} \\
FRIS & Fuzzy rough based filter method & {$[13]$} \\
OWA-FRPS-LU & New OWA-FRPS method based on the quality & - \\
& measure that takes into account both the lower & \\
OWA-FRPS-L & and upper approximation & \\
New OWA-FRPS method based on the quality & - \\
measure that takes into account the lower approx- & \\
OWA-FRPS-U & imation & \\
& New OWA-FRPS method based on the quality & - \\
measure that takes into account the upper approx- & \\
imation &
\end{tabular}


The reduction rate of the OWA-FRPS algorithms is about 30 percent, which is not as high as some of the evolutionary PS methods, but as the focus of our method is to improve the accuracy rather than reducing the storage needs, this result is of less importance.

The running time is of more interest to us. OWA-FRPS is slower than 6 other methods, but these methods have considerably lower accuracy rates. The running time of OWA-FRPS is shorter than the running times of the most accurate PS methods, so although OWA-FRPS is a wrapper and obtains excellent accuracy results, it does not come with the extra computational cost that wrapper PS methods typically have.

Table 6. Average results of the PS methods averaged over all datasets, ordered according to performance. Reduction is the ratio of removed instances, running time is given in seconds.

\begin{tabular}{ll|lr|lr} 
Accuracy & & Reduction & & Running Time \\
\hline OWA-FRPS-LUU & 0.8087 & CHC & 0.9681 & POP & 0.0083 \\
OWA-FRPS-L & 0.8053 & GGA & 0.9391 & MSS & 0.0297 \\
OWA-FRPS-U & 0.7948 & SSMA & 0.9356 & ModelCS & 0.0306 \\
RNG & 0.7901 & RNN & 0.9111 & MENN & 0.0474 \\
CHC & 0.7893 & RMHC & 0.9015 & FRIS & 0.0576 \\
ModelCS & 0.7892 & HMNEI & 0.5383 & AllKNN & 0.0580 \\
GGA & 0.7863 & MENN & 0.4723 & HMNEI & 0.0714 \\
AllKNN & 0.7837 & MSS & 0.4632 & OWA-FRPS-U & 0.1834 \\
SSMA & 0.7828 & OWA-FRPS-U & 0.3462 & OWA-FRPS-L & 0.1880 \\
FRIS & 0.7808 & AllKNN & 0.3377 & OWA-FRPS-LU & 0.2031 \\
RMHC & 0.7799 & OWA-FRPS-L & 0.3247 & RNG & 2.6473 \\
HMNEI & 0.7785 & OWA-FRPS-LU & 0.2766 & RNN & 6.3661 \\
POP & 0.7741 & RNG & 0.2323 & SSMA & 14.9963 \\
MENN & 0.7705 & ModelCS & 0.1152 & CHC & 16.3427 \\
MSS & 0.7674 & FRIS & 0.0799 & RMHC & 18.2093 \\
RNN & 0.7614 & POP & 0.0484 & GGA & 42.9252
\end{tabular}

\section{Conclusion and Future Work}

In this paper, we proposed a new PS method based on the OWA fuzzy rough set model, called OWA-FRPS. In order to select a subset of instances from the training set that improves the classification of the NN classifier, OWA-FRPS ranks the instances according to a OWA fuzzy rough measure, and then automatically selects a suitable threshold to select the final subset of instances. An experimental evaluation on several datasets shows that our method achieves accuracy rates that are better than those of state-of-the-art PS methods, and moreover, OWA-FRPS is considerably faster.

As future directions, we would like to expand the use of OWA-FRPS for other 
Table 7. Values of the statistics of the Friedman test and Holm post hoc procedure that compares OWA-FRPS-LU to the state-of the-art algorithms. The second column shows the Friedman ranks, the third column the Holm adjusted p-values.

\begin{tabular}{l|l|l} 
Method & Friedman Rank & Adj. p-value \\
\hline RNN & 10 & 0.003846 \\
MSS & 10 & 0.004167 \\
POP & 9 & 0.004545 \\
RMHC & 8 & 0.005 \\
FRIS & 8 & 0.005556 \\
MENN & 7.5 & 0.00625 \\
HMNEI & 7 & 0.007143 \\
SSMA & 7 & 0.008333 \\
AllKNN & 7 & 0.01 \\
GGA & 7 & 0.0125 \\
ModelCS & 7 & 0.016667 \\
CHC & 6.5 & 0.025 \\
RNG & 6 & 0.05 \\
OWA-FRPS-LU & 4 & -
\end{tabular}

classifiers like SVM and to improve OWA-FRPS for imbalanced datasets, that is, datasets for which one class is significantly more present than the other $[22$, 23].

\section{Acknowledgment}

This work was partially supported by the Spanish Ministry of Science and Technology under Project TIN2011-28488.

\section{References}

1. Cover, T., Hart, P.: Nearest neighbor pattern classification. IEEE Transactions on Information Theory 13(1) (1967) 21-27

2. García, S., Derrac, J., Cano, F.: Prototype selection for nearest neighbor classification: Taxonomy and empirical study. IEEE Transactions on Pattern Analysis and Machine Intelligence 34(3) (2012) 414-435

3. Cano, J., Herrera, F., Lozano, M.: Using evolutionary algorithms as instance selection for data reduction in kdd: an experimental study. IEEE Transactions on Evolutionary Computation 7(6) (2003) 561-575

4. Kuncheva, L., Jain, L.: Nearest neighbor classifier: Simultaneous editing and feature selection. Pattern Recognition Letters 20 (1999) 1149-1156

5. Kuncheva, L.: Editing for the k-nearest neighbors rule by a genetic algorithm. Pattern Recognition Letters 16(8) (1995) 809-814

6. García, S., Cano, J., Herrera, F.: A memetic algorithm for evolutionary prototype selection: A scaling up approach. Pattern Recognition 41 (2008) 2693-2709 
7. Skalak, D.: Prototype and feature selection by sampling and random mutation hill climbing algorithms. Machine Learning: Proceedings of the Eleventh International Conference (1994) 293-301

8. Sanchez, J., Pla, F., Ferri, F.: Prototype selection for the nearest neighbour rule through proximity graphs. Pattern Recognition Letters 18 (1997) 507-513

9. Tomek, I.: An experiment with the edited nearest-neighbor rule. IEEE Transactions on Systems, Man and Cybernetics 6(6) (1976) 448-452

10. Hattori, K., Takahashi, M.: A new edited k-nearest neighbor rule in the pattern classification problem. Pattern Recognition 32 (2000) 521-528

11. Cornelis, C., Jensen, R., Hurtado, G., Slezak, G.: Attribute selection with fuzzy decision reducts. Information Sciences 180(2) (2010) 209-224

12. Dubois, D., Prade, H.: Rough fuzzy sets and fuzzy rough sets. International Journal of General Systems 17 (1990) 191-209

13. Jensen, R., Cornelis, C.: Fuzzy-rough instance selection. Proceedings of the 19th International Conference on Fuzzy Systems (2010) 1776-1782

14. Cornelis, C., Verbiest, N., Jensen, R.: Ordered weighted average based fuzzy rough sets. Proceedings of the 5th international conference on Rough set and knowledge technology (2010) 78-85

15. Yager, R.: On ordered weighted averaging aggregation operators in multicriteria decisionmaking. IEEE Transactions on Systems, Man and Cybernetics 18 (1988) $183-190$

16. Marchiori, E.: Hit miss networks with applications to instance selection. Journal of Machine Learning Research 9 (2008) 997-1017

17. Brodley, C.: Recursive automatic bias selection for classifier construction. Machine Learning 20 (1995) 63-94

18. Barandela, R., Ferri, F., Sanchez, J.: Decision boundary preserving prototype selection for nearest neighbor classification. International Journal of Pattern Recognition and Artificial Intellgence 19 (2005) 787-806

19. Riquelme, J., Aguilar-Ruiz, J., Aguilar-Ruiz, J., Toro, M.: Finding representative patterns with ordered projections. Pattern Recognition 36(4) (2003) 1009-1018

20. Gates, G.: The reduced nearest neighbor rule. IEEE Transactions on Information Theory 18(3) (1972) 431-433

21. Derrac, J., García, S., Molina, D., Herrera, F.: A practical tutorial on the use of nonparametric statistical tests as a methodology for comparing evolutionary and swarm intelligence algorithms. Swarm and Evolutionary Computation 1(1) (2011) $3-18$

22. Ramentol, E., Caballero, Y., Bello, R., Herrera, F.: Smote-rsb*: A hybrid preprocessing approach based on oversampling and undersampling for high imbalanced data-sets using smote and rough sets theory. Knowledge and Information Systems (2011) 1-21

23. Ramentol, E., Verbiest, N., Bello, R., Caballero, Y., Cornelis, C., Herrera, F.: Smote-frst: A new resampling method using fuzzy rough set theory. In proceedings of the 10th International FLINS Conference on Uncertainty Modeling in Knowledge Engineering and Decision Making (2012) 800-805 